\title{
Analysis of Correlation and Total Power Radiometer Front-Ends Using Noise Waves
}

\author{
Ignasi Corbella, Member, IEEE, Francesc Torres, Member, IEEE, Adriano Camps, Senior Member, IEEE, \\ Nuria Duffo, Member, IEEE, Mercè Vall-llossera, Member, IEEE, Kimmo Rautiainen, Member, IEEE, \\ Manuel Martín-Neira, Member, IEEE, and Andreas Colliander, Member, IEEE
}

\begin{abstract}
A complete and systematic noise analysis of radiometer front-ends, including both total power and correlation measurements, is presented. The procedure uses the concepts of noise waves and $S$-parameters, widely used in microwave systems design and takes into account full noise characterization of receivers including mismatch effects. The general formulation is compatible with known total power radiometer analysis and is specially appropriate in correlation radiometers for which the effect of nonideal components, such as input isolators, is analyzed. Along with numerical simulations, simple formulas are given to compute the measured visibility in nonideal conditions. The analysis is validated using experimental results consisting of correlation measurements of four receivers placed inside an anechoic chamber. Good agreement between theoretical predictions and experimental data is observed.
\end{abstract}

Index Terms-Interferometric aperture synthesis radiometry, microwave radiometry, polarimetric radiometry, radiometer calibration.

\section{INTRODUCTION}

A CCURATE radiometer performance analysis requires good understanding of receivers' noise contribution from the antenna terminals to the detection circuitry. Total power radiometers analysis can be done in a quite straightforward manner through the use of relatively simple concepts such as receiver noise temperature and antenna temperature. But for instruments measuring cross-correlation between signals, such as aperture synthesis interferometric radiometers or polarimetric radiometers, the situation is much more complex. The measured total cross-correlation depends, not only on the cross-correlation of the input signals (which is the visibility or the third and fourth Stokes parameters, depending on the kind of radiometer), but also on the correlation among the different noise signals generated by the receivers themselves and propagated through the system via antenna coupling or by other means. A complete analysis of the radiometer behavior must

Manuscript received October 26, 2004; revised December 24, 2005. This work was supported in part by EADS-Casa Espacio and the European Space Agency in the frame of the MIRAS Demonstrator Pilot Project 3, in part by the Helsinki University of Technology (HUT-2D project), and in part by the Spanish MCYT and FEDER funds under Project TIC2002-04451-C02-01.

I. Corbella, F. Torres, A. Camps, N. Duffo, and M. Vall-llossera are with the Department of Signal Theory and Communications, Universitat Politècnica de Catalunya, 08034 Barcelona, Spain (e-mail: corbella@tsc.upc.es).

M. Martín-Neira is with the European Space Agency (ESA-ESTEC), 2200 Noordwijk, The Nederlands (e-mail: Manuel.Martin-Neira@esa.int).

A. Colliander and K. Rautiainen are with the Helsinki University of Technology, Espoo FIN-02015 HUT, Finland (e-mail:kimmo@avasun.hut.fi).

Digital Object Identifier 10.1109/TGRS.2005.847912

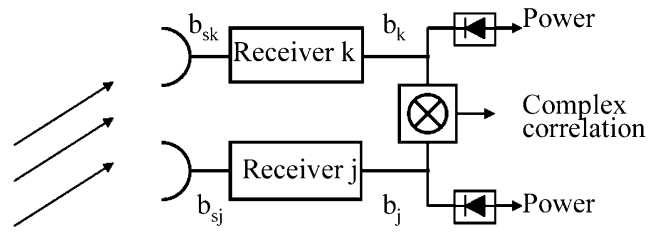

Fig. 1. Schematic block diagram of a correlation radiometer front-end.

include the interactions and mismatches of all the components forming the front-end, in particular the low-noise amplifiers and other radio-frequency subsystems.

The formalism presented in this paper includes all contributions to the measured cross-correlation and total power. The approach uses well-established concepts of microwave systems characterization, such as S-parameters and noise waves, and has the advantage of unifying the key parameters of total power radiometers, polarimetric radiometers, and interferometric radiometers in a single formulation. With this approach the signals responsible for the total power measurements and those of correlation are clearly and rigorously identified and defined in a consistent way.

The formulation is validated through the analysis of experimental data. This consists of correlation and total power measurements of a set of four receivers placed inside an anechoic chamber. Measurements show that there exists a residual offset that depends on the antenna coupling, and this is attributed to mismatch between antennas and receivers. Computer simulations based on the theoretical analysis predict these measured results and give tools to improve receivers' design in order to minimize this effect or others.

\section{RADIOMETER FRONT-END}

A general block diagram of a correlation and total power radiometer front-end is depicted in Fig. 1. It may represent a single baseline of an aperture synthesis radiometer or a polarimetric radiometer. In the first case, the antennas are physically different while in the second case the drawing represents both outputs of the same dual-polarization antenna. In both cases the fundamental measurements are the total power and the cross-correlation of the output signals. In aperture synthesis radiometry the cross-correlation is related to the visibility function while in polarimetric radiometry it relates to the third and fourth Stokes parameters. In this paper the name visibility will be used in general for correlation products, although a better name such as correlation temperature could be also used. The limiting case in which there is a single receiver represents a standard total power radiometer. 


\section{A. Basic Definitions}

To include the contribution of the receivers, it is convenient to extend the concepts of antenna and system temperature, commonly used in radiometry, also to the correlation product or visibility. Note that the Rayliegh-Jeans approximation is implicitly assumed, so that power is proportional to temperature. With reference to Fig. 1 the following definitions are here proposed.

- The antenna visibility $(\mathcal{V})$ and the antenna temperature $\left(T_{a}\right)$ are related respectively to the spectral cross-power and power densities of the signals coming from the external source

$$
\begin{aligned}
\overline{b_{s k}(f) b_{s j}^{*}(f)} & =k \mathcal{V}_{k j} \\
\overline{\left|b_{s k}(f)\right|^{2}} & =k T_{a k} \cdot(2)
\end{aligned}
$$

- The system visibility $\left(\mathcal{V}_{\text {sys }}\right)$ and the System temperature $\left(T_{\text {sys }}\right)$ are defined similarly but using the total signals, both from the external source and those generated in the receivers themselves referred to the input ports

$$
\begin{aligned}
\overline{b_{k}(f) b_{j}^{*}(f)} & =k H_{k}(f) H_{j}^{*}(f) \mathcal{V}_{\mathrm{sys}_{k j}} \\
\overline{\left|b_{k}(f)\right|^{2}} & =k\left|H_{k}(f)\right|^{2} T_{\text {sys }_{k}}
\end{aligned}
$$

where $k$ is the Boltzmann constant, $H(f)$ stands for the frequency response of the receivers, and the overbar denotes statistical expectation. The precise formulation of these equations, especially when mismatch between antenna and receiver is considered is given in Sections II-B-II-E.

\section{B. Correlation Matrices}

Fig. 2 shows a more detailed model of a typical front-end. The receivers are modeled as active networks characterized by their S-parameters $S^{(k)}, k$ denoting the receiver number. Since they always include high-gain amplifiers, $\left|S_{12}^{(k)}\right| \ll\left|S_{21}^{(k)}\right|$, in practice it can be assumed that $S_{12}^{(k)} \approx 0$ although this is not a restriction for the present analysis. The signals $b_{k 2}$ and $b_{j 2}$ in Fig. 2 are defined as the output waves of the receivers when these ones are loaded by the reference impedance (zero reflection coefficient) at absolute zero temperature. ${ }^{1}$ With this definition the output wave of receiver $k$ is

$$
b_{k 2}=S_{21}^{(k)} b_{k}+c_{k 2}
$$

where $c_{k 2}$ is the noise signal generated by the receiver at its output port [1] (see the Appendix for a more accurate definition). In order to refer the output wave to the input port, this is conveniently rewritten as

$$
b_{k 2}=S_{21}^{(k)}\left(b_{k}+\frac{c_{k 2}}{S_{21}^{(k)}}\right)=S_{21}^{(k)} b_{k}^{\prime}
$$

where $b_{k}^{\prime}$ is defined as the equivalent total input wave. It contains contributions of the signal coming from the antennas and of the noise generated by the receivers taking into account also the mismatch at the antenna-receiver interface. The power and

\footnotetext{
${ }^{1}$ This is a formal definition. In practice the contribution of the thermal noise generated by the load and reflected by the receiver output port is negligible due to the usual high gain of the receiver
}

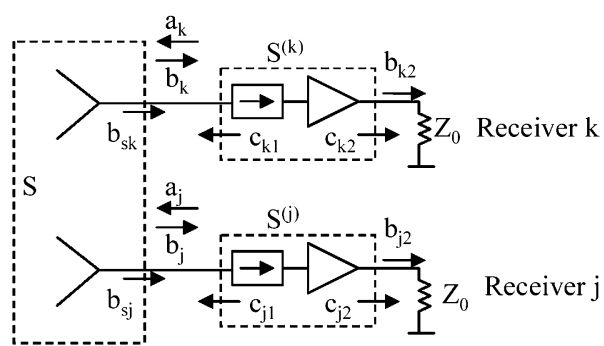

Fig. 2. Electrical model of a baseline. The receivers are active two-ports generating partially correlated waves $\left(c_{k 1}\right.$ and $\left.c_{k 2}\right)$. The set of both antennas pointing to a thermal source is modeled as a passive two-port with given S-parameters.

cross-power densities of these equivalent total input waves are conveniently used to make a precise definition of the system temperature and visibility respectively

$$
\overline{b_{k}^{\prime} b_{j}^{\prime *}}=k \mathcal{V}_{\text {sys }_{k j}} \quad \overline{\left|b_{k}^{\prime}\right|^{2}}=k T_{\text {sys }_{k}}
$$

from which the power and cross-power densities of the output signals (6) become

$$
\overline{b_{k 2} b_{j 2}^{*}}=k S_{21}^{(k)} S_{21}^{(j) *} \mathcal{V}_{\mathrm{sys}_{k j}} \quad \overline{\left|b_{k 2}\right|^{2}}=k\left|S_{21}^{(k)}\right|^{2} T_{\mathrm{sys}_{k}}
$$

When comparing these with (3) and (4) it comes clear that the definitions for $T_{\text {sys }}$ and $\mathcal{V}_{\text {sys }}$ of (7) are such that the receivers frequency response is always the transmission $S$-parameter independently of the matching conditions of the receivers. This is not an approximation, but a consequence of the proposed definition of system temperature. Note that the available output power is $\left|b_{k 2}\right|^{2} /\left(1-\left|\Gamma_{\text {out }}\right|^{2}\right)$ where $\Gamma_{\text {out }}$ is the output reflection coefficient. A more detailed discussion on this topic is given in Section II-C1. From (7), the correlation matrix of the equivalent total input waves is

$$
\overline{\mathbf{b}^{\prime} \mathbf{b}^{\prime \dagger}}=k\left[\begin{array}{cc}
T_{\text {sys }_{k}} & \mathcal{V}_{\text {sys }_{k j}} \\
\mathcal{V}_{\text {sys }_{j k}} & T_{\text {sys }_{j}}
\end{array}\right]=k \mathbf{V}_{\text {sys }}
$$

where the dagger indicates Hermitian transpose operation, the boldface letters are used for matrices or vectors and $\mathbf{b}^{\prime}$ is the column vector of equivalent total input waves.

As indicated in (1) and (2), $b_{s k}$ and $b_{s j}$ in Figs. 1 or 2 are waves coming only from the external sources and used to define the antenna temperature and the antenna visibility. ${ }^{2}$ They are here defined in a similar way as $b_{k 2}$ and $b_{j 2}$, i.e., as the noise waves on the reference impedance at absolute zero. The visibility matrix is defined using the correlation matrix of these waves

$$
\overline{\mathbf{b}_{\mathbf{s}} \mathbf{b}_{\mathbf{s}}^{\dagger}}=k\left[\begin{array}{ll}
T_{a k} & \mathcal{V}_{k j} \\
\mathcal{V}_{j k} & T_{a j}
\end{array}\right]=k \mathbf{V}
$$

It should be noted that $T_{a k}$ and $\mathcal{V}_{k j}$ are the antenna noise temperature and frequency-domain visibility exactly as defined in [2, eqs. (6) and (7)]. In Sections II-C-II-E the relation between the matrices of system visibility and antenna visibility will be obtained in the general case and particularized for different cases.

${ }^{2}$ Lossless antennas are assumed at this stage. Antenna losses are addressed in Section II-E 


\section{Front-End Analysis}

The incident and reflected waves at the antenna ports of Fig. 2(a) and (b), respectively, are mutually related by the antenna system $\mathbf{S}$-parameters $\mathbf{S}$ and also by the receivers input reflection coefficients. Using standard matrix algebra, the following expression for the vector of equivalent total input waves is found:

$$
\mathrm{b}^{\prime}=\tilde{\mathbf{S}} \mathbf{c}_{1}+\Lambda \mathrm{b}_{\mathrm{s}}+\mathrm{c}_{2}^{\prime}
$$

where $\boldsymbol{\Lambda}=\left(\mathbf{I}-\mathbf{S} \boldsymbol{\Gamma}_{\mathbf{R}}\right)^{-1}, \tilde{\mathbf{S}}=\boldsymbol{\Lambda} \mathbf{S}, \mathbf{I}$ is the identity matrix and

$$
\boldsymbol{\Gamma}_{\mathbf{R}}=\left[\begin{array}{cc}
S_{11}^{(k)} & 0 \\
0 & S_{11}^{(j)}
\end{array}\right] \text {. }
$$

The wave vectors appearing in the right-hand side of (11) are defined as follows: $\mathbf{c}_{\mathbf{1}}$ contains the noise generated by the receivers from their input ports, $\mathbf{b}_{\mathbf{s}}$ the signals coming from the external target and $\mathbf{c}_{2}^{\prime}$ the noise generated by the receivers at their output port referred to the input. The system visibility matrix, is now easily computed by introducing (11) into (9)

$$
\mathbf{V}_{\mathrm{sys}}=\boldsymbol{\Lambda} \mathbf{V} \Lambda^{\dagger}+\tilde{\mathbf{S}} \mathbf{T}_{\mathbf{r}} \tilde{\mathbf{S}}^{\dagger}+\tilde{\mathbf{S}} \mathbf{T}_{\mathbf{c}}+\mathbf{T}_{\mathbf{c}}^{\dagger} \tilde{\mathbf{S}}^{\dagger}+\mathbf{T}_{\mathbf{R}}
$$

where still lossless antennas are considered, it has been assumed that $\overline{\mathbf{c}_{\mathbf{1}} \mathbf{b}_{\mathrm{s}}^{\dagger}}=\overline{\mathbf{b}_{\mathrm{s}} \mathbf{c}_{\mathbf{1}}^{\dagger}}=\overline{\mathbf{c}_{\mathbf{2}}^{\prime} \mathbf{b}_{\mathrm{s}}^{\dagger}}=\overline{\mathbf{b}_{\mathrm{s}} \mathbf{c}_{\mathbf{2}}^{\prime \dagger}}=\mathbf{0}$ and the following definitions have been used:

$$
\begin{aligned}
& \overline{\mathbf{c}_{\mathbf{1}} \mathbf{c}_{\mathbf{1}}^{\dagger}}=k\left[\begin{array}{cc}
T_{r k} & 0 \\
0 & T_{r j}
\end{array}\right]=k \mathbf{T}_{\mathbf{r}} \\
& \overline{\mathbf{c}_{\mathbf{1}} \mathbf{c}_{\mathbf{2}}^{\prime \dagger}}=k\left[\begin{array}{cc}
T_{c k} & 0 \\
0 & T_{c j}
\end{array}\right]=k \mathbf{T}_{\mathbf{c}} \\
& \overline{\mathbf{c}_{\mathbf{2}}^{\prime} \mathbf{c}_{\mathbf{2}}^{\prime \dagger}}=k\left[\begin{array}{cc}
T_{R k} & 0 \\
0 & T_{R j}
\end{array}\right]=k \mathbf{T}_{\mathbf{R}}
\end{aligned}
$$

where $T_{r}, T_{c}$, and $T_{R}$ are the three receiver equivalent noise temperatures defined in [3]. Since they play an important role in the following discussions, their definition and properties are given in the Appendix. As stated there, in [1] it is shown that $T_{R}$ is the standard definition of receiver noise temperature for zero source reflection coefficient.

Equation (13) is the general formula relating the matrix of system visibility to the matrix of antenna visibility taking into account all receivers' contributions. Several special situations will be considered in Sections II-C1-II-C4.

1) Total Power Radiometers: In the case of having a single antenna and receiver, (13) reduces to the scalar equation

$$
T_{\mathrm{sys}}=|\Lambda|^{2} T_{a}+|\Lambda|^{2}\left|\Gamma_{a}\right|^{2} T_{r}+2 \Re e\left[\Lambda \Gamma_{a} T_{c}\right]+T_{R}
$$

where $\Gamma_{a}$ is the antenna reflection coefficient and

$$
\Lambda=\frac{1}{1-S_{11} \Gamma_{a}}
$$

where $S_{11}$ is the input reflection coefficient of the receiver (there is no need of superscript here). In the Appendix it is shown that (17) can be written as

$$
\frac{\left|S_{21}\right|^{2} T_{\mathrm{sys}}}{1-\left|\Gamma_{\mathrm{out}}\left(\Gamma_{a}\right)\right|^{2}}=G_{\mathrm{av}}\left(\Gamma_{a}\right)\left(\frac{T_{a}}{1-\left|\Gamma_{a}\right|^{2}}+T_{R}\left(\Gamma_{a}\right)\right)
$$

where $S_{21}$ is the transmission S-parameter of the receiver, $G_{\mathrm{av}}\left(\Gamma_{a}\right)$ its available power gain, $\Gamma_{\text {out }}\left(\Gamma_{a}\right)$ its output reflection coefficient, and $T_{R}\left(\Gamma_{a}\right)$ its equivalent noise temperature, all three computed for a source reflection coefficient equal to $\Gamma_{a}$. The term $T_{a} /\left(1-\left|\Gamma_{a}\right|^{2}\right)$ is recognized as the antenna temperature defined in terms of available power. According to (8) and the definition of output waves, the left-hand side of the above equation is the available output power, so (19) is consistent with a definition of system and antenna temperature in terms of available power as long as the available power gain is used. Moreover, by introducing the expression of the available power (40) into (19), the following relation between two definitions of system temperature is obtained:

$$
T_{\mathrm{sys}}=\frac{1-\left|\Gamma_{a}\right|^{2}}{\left|1-S_{11} \Gamma_{a}\right|^{2}} T_{\mathrm{sys}}^{\mathrm{av}}
$$

where $T_{\text {sys }}$ is the definition used in this paper and $T_{\text {sys }}^{\mathrm{av}}$ is a definition based on available power $T_{\mathrm{sys}}^{\mathrm{av}}=T_{a}^{\mathrm{av}}+T_{R}\left(\Gamma_{a}\right)$ where $T_{a}^{\mathrm{av}}$ is the definition of antenna temperature based on available power. Both definitions coincide when $\Gamma_{a}=0$.

While definitions based on available power may be convenient for total power radiometers, when correlation measurements are considered their utility is reduced. The complex waves used to define the correlation product must be clearly identified and the best approach is using the waves $b_{s}$, since they are uniquely and rigorously defined for any number of antennas.

2) Constant Source: Considering now the case of two or more antennas, if the output source is homogeneous with physical temperature $T_{0}$, as for example if the baseline is placed inside an anechoic chamber, application of the Bosma theorem [4] in (10) gives $\mathbf{V}=T_{0}\left(\mathbf{I}-\mathbf{S S}^{\dagger}\right)$ which, substituted into (13), results in

$$
\mathbf{V}_{\mathrm{sys}}=T_{0} \mathbf{\Lambda} \mathbf{\Lambda}^{\dagger}+\tilde{\mathbf{S}}\left(\mathbf{T}_{\mathbf{r}}-T_{0} \mathbf{I}\right) \tilde{\mathbf{S}}^{\dagger}+\tilde{\mathbf{S}} \mathbf{T}_{\mathbf{c}}+\mathbf{T}_{\mathbf{c}}^{\dagger} \tilde{\mathbf{S}}^{\dagger}+\mathbf{T}_{\mathbf{R}}
$$

Note that the term $\mathbf{T}_{\mathbf{r}}-T_{0} \mathbf{I}$ vanishes if the equivalent temperature of the receivers at their input port equals the source physical temperature.

3) Ideal Input Isolators: The S-parameters and correlation matrices of an ideal isolator at physical temperature $T_{\mathrm{ph}}$ are

$$
\mathbf{S}_{I}^{(k)}=\left[\begin{array}{ll}
0 & 0 \\
1 & 0
\end{array}\right] \quad \mathbf{C}_{I}^{(k)}=k T_{\mathrm{ph}}\left[\begin{array}{ll}
1 & 0 \\
0 & 0
\end{array}\right]
$$

Then, if the first component in the receiver chain is an ideal isolator it comes out that $S_{11}^{(k)}=0$ and from (12) $\boldsymbol{\Gamma}_{\mathbf{R}}=0$, so $\boldsymbol{\Lambda}=\mathbf{I}$ and $\tilde{\mathbf{S}}=\mathbf{S}$. Moreover, the input-output correlation temperature (15) vanishes $\left(\mathbf{T}_{\mathbf{c}}=\mathbf{0}\right)$, and the input noise temperature (14) becomes equal to the isolator physical temperature $T_{r k}=T_{\mathrm{ph}_{\mathrm{k}}}$. Then (13) becomes

$$
\mathbf{V}_{\text {sys }}=\mathbf{V}+\mathbf{S} \mathbf{T}_{\mathbf{p h}} \mathbf{S}^{\dagger}+\mathbf{T}_{\mathbf{R}}
$$

where $\mathbf{T}_{\mathrm{ph}}$ is a diagonal matrix containing the physical temperature of the isolators. In the special case of having a constant source at $T_{0}(21)$ reduces to $\mathbf{V}_{\text {sys }}=T_{0} \mathbf{I}+\tilde{\mathbf{S}}\left(\mathbf{T}_{\mathbf{p h}}-T_{0} \mathbf{I}\right) \tilde{\mathbf{S}}^{\dagger}+$ $\mathbf{T}_{\mathbf{R}}$ which gives the result consistent with [2] that, when the receivers are at thermodynamic equilibrium inside an anechoic chamber, the system temperature is $T_{0}+T_{R k}$ and the system visibility vanishes. 
In general, from (23), the difference between both visibility matrices is $\mathbf{V}_{\text {sys }}-\mathbf{V}=\mathbf{S} \mathbf{T}_{\mathbf{p h}} \mathbf{S}^{\dagger}+\mathbf{T}_{\mathbf{R}}$, which is expanded to

$$
\begin{aligned}
T_{\text {sys }_{k}}-T_{a k} & =\left|S_{k k}\right|^{2} T_{\mathrm{ph} k}+\left|S_{k j}\right|^{2} T_{\mathrm{ph} j}+T_{R k} \\
\mathcal{V}_{\mathrm{sys}_{k j}}-\mathcal{V}_{k j} & =S_{k k} S_{j k}^{*} T_{\mathrm{ph} k}+S_{k j} S_{j j}^{*} T_{\mathrm{ph} j .}
\end{aligned}
$$

Note that if the antennas are well matched and there is no coupling between them $\left(S_{k j}=0\right.$ for $\left.k, j=1,2\right)$, the system temperature becomes equal to the antenna temperature plus the receiver noise temperature and the system visibility equal to the antenna visibility, as it is expected.

4) Imperfect (But Good) Input Isolator: If the input isolator is not perfect, the complete (13) should be considered. However, if both the isolators and the antennas are reasonably well matched, $\boldsymbol{\Lambda}$ defined just before (12) can be approximated as $\Lambda_{k k} \approx 1$ and $\Lambda_{k j} \approx S_{k j} S_{11}^{(j)}$ and also $\tilde{\mathbf{S}} \approx \mathbf{S}$. Introducing these approximations in (13), two equations similar to (24) can be found for this case

$$
\begin{aligned}
T_{\mathrm{sys}_{k}}-T_{a k}= & \left|S_{k k}\right|^{2} T_{r k}+\left|S_{k j}\right|^{2} T_{r j}+2 \Re e\left[S_{k k} T_{c k}\right]+T_{R k} \\
\mathcal{V}_{\mathrm{sys}_{k j}}-\mathcal{V}_{k j}= & S_{k k} S_{j k}^{*} T_{r k}+S_{k j} S_{j j}^{*} T_{r j}+S_{j k}^{*} S_{11}^{(k) *} T_{a k} \\
& +S_{k j} S_{11}^{(j)} T_{a j}+S_{j k}^{*} T_{c k}^{*}+S_{k j} T_{c j} .
\end{aligned}
$$

The main differences between (25) and (24) are that the physical temperatures now have been substituted by the input noise temperatures of the receivers and extra terms have appeared, which are significant in the visibility and negligible in the system temperature.

In (25) the input and correlation temperatures $\left(T_{r k}\right.$ and $\left.T_{c k}\right)$ correspond to the total receiver, which is formed by the cascade of the isolator and the amplifier (Fig. 2). The noise temperatures of a cascade of networks can be computed as a function of the ones of the individual components by using the formulas given in the Appendix. Assuming that both the low-noise amplifier and the isolator are well matched and that the isolation is fairly high, the following approximated equations can be used:

$$
\begin{aligned}
& T_{r k} \approx T_{r k_{I}} \approx T_{\mathrm{ph}_{k}} \\
& T_{c k} \approx T_{c k_{I}}=-T_{\mathrm{ph}_{k}}\left(\frac{S_{11_{I}}^{(k)}+S_{12_{I}}^{(k)} S_{22_{I}}^{(k) *}}{S_{21_{I}}^{(k) *}}\right)
\end{aligned}
$$

where $T_{\mathrm{ph}_{k}}$ is the physical temperature of the isolator and the subscript $I$ indicates that the corresponding parameter is that of the isolator.

Subtracting (25) from (24), the visibility offset due to imperfect isolator $\Delta \mathcal{V}$ is obtained. Using (26) and assuming that both receivers are identical, the following simplified expression is obtained:

$$
\Delta \mathcal{V}=2 \Re e\left[S_{j k}^{*}\left(T_{a k} S_{11}^{(k) *}+T_{c k_{I}}^{*}\right)\right]
$$

where $T_{c k_{I}}$ is given by (27). This equation shows that the system visibility offset due to antenna coupling is directly proportional to the amplitude of the antenna coupling coefficient $\left(\left|S_{j k}\right|\right)$ and has a sinusoidal dependence with its phase. Its concrete value depends then on the transmission line lengths connecting the antenna and the isolator. Moreover, its maximum amplitude depends on the phase combination of the two complex summands of the formula wich include the receiver input reflection coefficent and the isolator correlation temperature. It is important to
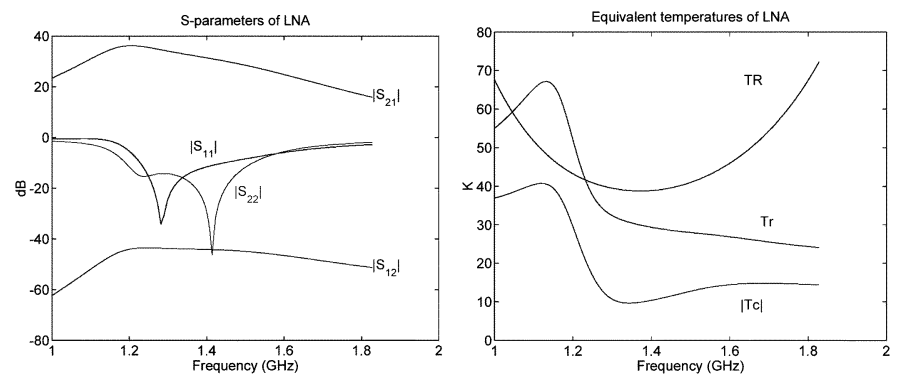

Fig. 3. Low-noise amplifier characteristics. (Left) S-parameters. (Right) Equivalent noise temperatures.
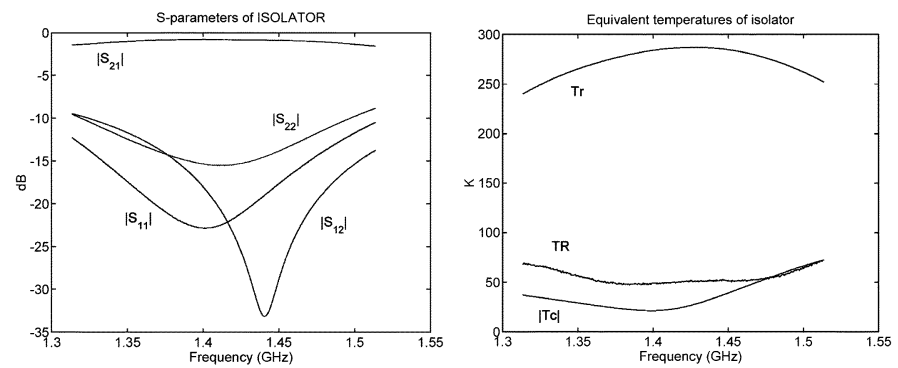

Fig. 4. Isolator characteristics. (Left) S-parameters. (Right) Equivalent noise temperatures.

point out that in this formula, which assumes fairly good isolator, the noise temperatures of the LNA do not appear. This is an important feature, since these parameters are always difficult to measure.

\section{Numerical Validation Based on Experimental Data}

To validate the above equation, numerical simulations have been performed using available measured data of a low-noise amplifier (LNA) and an isolator. The the LNA was designed and manufactured by MIER Comunicaciones S.A. (Spain) as part of the receivers of the radiometer MIRAS [5], single payload of the European Space Agency's Soil Moisture and Ocean Salinity mission [6]. Its S-parameters and noise temperatures as a function of frequency are shown in Fig. 3. At the center frequency $f_{0}=1413.5 \mathrm{MHz}$ the gain is about $30 \mathrm{~dB}$, the input return loss $11 \mathrm{~dB}$, and the noise temperatures are $T_{r k}=29.13 \mathrm{~K}$, $T_{c k}=10.67 \angle 23.74^{\circ} \mathrm{K}$ and $T_{R k}=39 \mathrm{~K}$.

The isolator is a commercial unit, and its S-parameters and noise temperatures are shown in Fig. 4. The S-parameters are measured and the noise temperatures have been computed directly from them and from the physical temperature using the formulas of the Appendix. At the center frequency the insertion loss is $0.8 \mathrm{~dB}$, the input return loss $22 \mathrm{~dB}$ and the isolation $21 \mathrm{~dB}$.

The system visibility that would be measured by a baseline placed inside an anechoic chamber at the same physical temperature as the receivers has been computed both exactly (21) and using the approximated formula (28). For this particular simulation an antenna coupling of $20 \mathrm{~dB}$ was considered. Fig. 5 shows the comparison between both simulations as a function of the phase of the antenna coupling coefficient. As can be seen, the agreement, especially in terms of maximum values of visibility, is very good, thus validating the use of the approximation for estimating the maximum offset. The maximum value of the correlation is about $2 \mathrm{~K}$ and the computed system temperature 


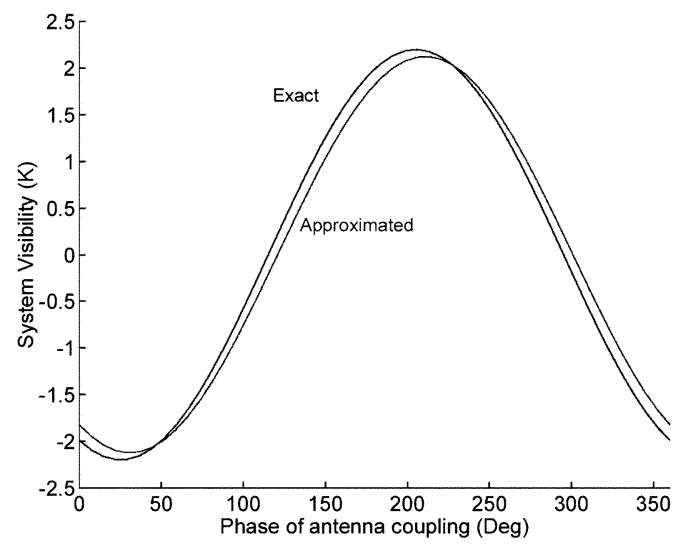

Fig. 5. System visibility of a baseline in an anechoic chamber using exact and approximate formulas.

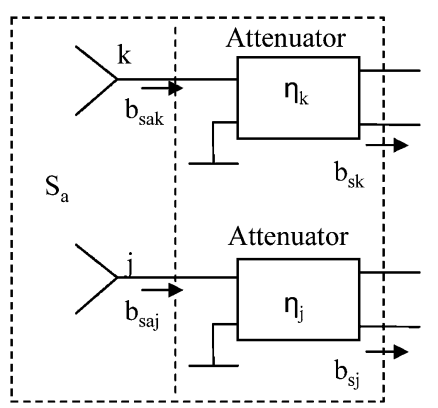

Fig. 6. Equivalent model of a baseline with lossy antennas.

was about $400 \mathrm{~K}$, so the maximum normalized correlation that would be measured is about $|\mu| \approx 2 / 400=51 \times 10^{-4}$. This is consistent with the experimental results presented in Section III.

\section{E. Antenna Losses}

The standard model to take into account antenna losses consists of cascading a lossless antenna to an ideal attenuator with S-parameters given by $S_{11}=S_{22}=0$ and $S_{12}=S_{21}=\sqrt{\eta}$ where $\eta$ is the antenna efficiency. It should be pointed out that this model is general and includes even a configuration consisting of a physical unmatched antenna followed by a lossy matching network. In this case, however, the S-parameters of the matching network are not those of the attenuator that emulates the antenna efficiency. The model tells that the whole system "actual antenna + matching circuit" is equivalent to a lossless antenna followed by an ideal attenuator.

Using this model in both antennas, a baseline can be modeled as shown in Fig. 6. To use the standard denomination when losses are present (as, for example, in [7, p. 208]), the antenna temperature and antenna visibility as seen by the receiver will be denoted by $T_{a}^{\prime}$ and $\mathcal{V}^{\prime}$ respectively while the same magnitudes referred to the lossless antennas will be unprimed. With this convention, and to be consistent with the different definitions of this paper, the following definitions apply to the waves in Fig. 6:

$$
\begin{aligned}
k T_{a k}^{\prime}=\overline{\left|b_{s k}\right|^{2}} & k T_{a k}=\overline{\left|b_{s a k}\right|^{2}} \\
k \mathcal{V}_{k j}^{\prime} & =\overline{b_{s k} b_{s j}^{*}} \quad k \mathcal{V}_{k j}=\overline{b_{s a k} b_{s a j}^{*}} .
\end{aligned}
$$

When antenna losses are included, the matrix $\mathbf{V}$ in (13) should be replaced by $\mathbf{V}^{\prime}$, defined in the same way as $\mathbf{V}$ in
(10) but using $T_{a}^{\prime}$ and $\mathcal{V}_{k j}^{\prime}$ instead of $T_{a}$ and $\mathcal{V}_{k j}$. To refer the correlation matrix to the "lossless" antenna temperature and antenna visibility, it is only needed to put $\mathbf{V}^{\prime}$ in terms of $\mathbf{V}$. This can be done quite straightforward if one is aware that the setup of Fig. 6 is exactly the same as that of Fig. 2 in which the whole receivers are now replaced by the attenuators. The equivalent noise temperatures of an attenuator with $S_{11}=S_{22}=0$ and $S_{12}=S_{21}=\sqrt{\eta}$ are $T_{r}=T_{\mathrm{ph}}(1-\eta), T_{c}=0$ and $T_{R}=T_{\mathrm{ph}}(1 / \eta-1)$, so the correlation matrix of the equivalent signals at the attenuators inputs comes directly from (13). The antenna visibility and antenna temperatures at receiver inputs are obtained then using (8) from this correlation matrix. After some matrix manipulation, this leads to

$$
\mathbf{V}^{\prime}=\boldsymbol{\Upsilon}^{1 / 2} \mathbf{V} \boldsymbol{\Upsilon}^{1 / 2}+\mathbf{S} \mathbf{T}_{\mathbf{p}}\left(\boldsymbol{\Upsilon}^{-1}-\mathbf{I}\right) \mathbf{S}^{\dagger}+\mathbf{T}_{\mathbf{p}}(\mathbf{I}-\boldsymbol{\Upsilon})
$$

where $\boldsymbol{\Upsilon}$ and $\mathbf{T}_{\mathbf{p}}$ are diagonal matrices containing respectively the antenna efficiencies and the physical temperatures of the antennas

$$
\boldsymbol{\Upsilon}=\left[\begin{array}{cc}
\eta_{k} & 0 \\
0 & \eta_{j}
\end{array}\right] \quad \mathbf{T}_{\mathbf{p}}=\left[\begin{array}{cc}
T_{\mathrm{ph} k} & 0 \\
0 & T_{\mathrm{ph} j}
\end{array}\right]
$$

and the $S$-parameters are those defined at the external antenna terminals, that is including the losses. Equation (30) can be fully understood if some special situations are considered.

- For lossless antennas $\boldsymbol{\Upsilon}=\mathbf{I}$, so that $\mathbf{V}^{\prime}=\mathbf{V}$ as expected.

- If the antennas are perfectly matched and there is no coupling between them, then $\mathbf{S}=\mathbf{0}$ and the equation is reduced to $\mathbf{V}^{\prime}=\boldsymbol{\Upsilon}^{1 / 2} \mathbf{V} \boldsymbol{\Upsilon}^{1 / 2}+\mathbf{T}_{\mathbf{p}}(\mathbf{I}-\boldsymbol{\Upsilon})$ which is easily expanded to

$$
T_{a k}^{\prime}=\eta_{k} T_{a k}+T_{\mathrm{ph} k}\left(1-\eta_{k}\right) \quad \mathcal{V}_{k j}^{\prime}=\mathcal{V}_{k j} \sqrt{\eta_{k} \eta_{j}}
$$

and they are consistent with [7, eq. (4.16)] and with the fact that the noise generated by the ohmic losses in each antenna is uncorrelated with each other.

- Finally, if the antennas are placed inside an anechoic chamber at a constant temperature $T_{0}$ equal to the physical temperature of the antennas, then $\mathbf{T}_{\mathbf{p}}=T_{0} \mathbf{I}$ and $\mathbf{V}=T_{0}\left(\mathbf{I}-\mathbf{S}_{\mathbf{a}} \mathbf{S}_{\mathbf{a}}^{\dagger}\right)$ where $\mathbf{S}_{\mathbf{a}}$ are the S-parameters of the "lossless part" of the antenna. Taking into account that $\mathbf{S}=\boldsymbol{\Upsilon}^{1 / 2} \mathbf{S}_{\mathbf{a}} \boldsymbol{\Upsilon}^{1 / 2}$ it is straightforward to get the result: $\mathbf{V}^{\prime}=T_{0}\left(\mathbf{I}-\mathbf{S} \mathbf{S}^{\dagger}\right)$, consistent with the Bosma theorem.

The final step is introducing (30) into (13) to get the correlation matrix of the equivalent signals at the receivers input in terms of the antenna visibility and antenna temperature. The result is that (13) is still valid as long as the following substitutions are made:

$$
\begin{aligned}
\mathbf{V} & \rightarrow \boldsymbol{\Upsilon}^{1 / 2} \mathbf{V} \mathbf{\Upsilon}^{1 / 2} \\
\mathbf{T}_{\mathbf{r}} & \rightarrow \mathbf{T}_{\mathbf{r}}+\mathbf{T}_{\mathbf{p}}\left(\boldsymbol{\Upsilon}^{-1}-\mathbf{I}\right) \\
\mathbf{T}_{\mathbf{R}} & \rightarrow \mathbf{T}_{\mathbf{R}}+\boldsymbol{\Lambda} \mathbf{T}_{\mathbf{p}}(\mathbf{I}-\boldsymbol{\Upsilon}) \boldsymbol{\Lambda}^{\dagger}
\end{aligned}
$$

This result is not surprising. It can be summarized as saying that when losses are present the antenna temperature and antenna visibility at the receivers input are those given by (32) and the contribution of the noise generated by the antenna losses must be added to the equivalent input and output temperatures of the receivers.

\section{EXPERIMENTAL RESULTS}

Correlation measurements were carried out at the Helsinki University of Technology (HUT) using a setup consisting of 4 


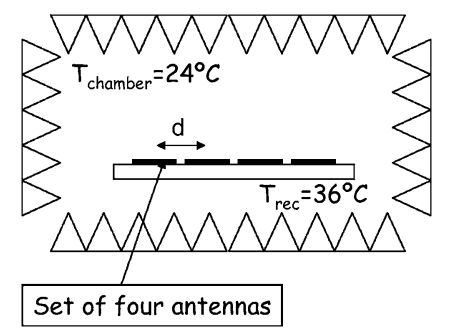

Fig. 7. Setup of four antennas inside an anechoic chamber used in the correlation measurements.

TABLE I

AnTENna Coupling MEASurement

\begin{tabular}{|c|c|c|c|c|}
\hline \multicolumn{2}{|c|}{$\begin{array}{l}\text { Test antenna position \#, } \\
\text { receiver polarisation }\end{array}$} & & & \\
\hline \multirow{3}{*}{$\begin{array}{l}\# 1, \text { Vpol } \\
\# 1, \text { Hpol }\end{array}$} & \multirow{2}{*}{$\begin{array}{l}\text { coupling to: } \\
\text { Amplidude }[\mathrm{dB}]\end{array}$} & rec2 & rec3 & $\overline{\text { rec4 }}$ \\
\hline & & -22.5 & -35.5 & -42.4 \\
\hline & \multirow{3}{*}{ coupling to: } & $\frac{-23.6}{\text { rec1 }}$ & $\begin{array}{c}-43.4 \\
\text { rec3 }\end{array}$ & $\frac{-49.1}{\text { rec } 4}$ \\
\hline \multirow{2}{*}{$\begin{array}{l}\# 2, \text { Vpol } \\
\# 2, \mathrm{Hpol}\end{array}$} & & -22.9 & -23.4 & -32.7 \\
\hline & & -23.1 & -20.7 & -45.8 \\
\hline \multirow{3}{*}{$\begin{array}{l}\text { \#3, Vpol } \\
\# 3, \text { Hpol }\end{array}$} & \multirow[t]{3}{*}{ coupling to: } & rec1 & rec2 & rec4 \\
\hline & & -36.0 & -23.7 & -22.4 \\
\hline & & -46.8 & -23.6 & -23.6 \\
\hline \multirow{3}{*}{$\# 4$, Vpol } & \multirow[t]{3}{*}{ coupling to: } & rec1 & rec2 & rec3 \\
\hline & & -44.6 & -34.8 & -24.0 \\
\hline & & -51.3 & -51.6 & -26.7 \\
\hline
\end{tabular}

antennas mounted on a linear structure and introduced in an anechoic chamber (Fig. 7). This hardware is a prototype section of the HUT 2-D aperture synthesis radiometer [8].

The coupling between antennas was first measured with a vector network analyzer using a test antenna as a sending unit. The test antenna was placed in each receiver location replacing that specific receiver. Table I shows the results of the measurements. As it can be seen, the coupling between adjacent antennas ranges from $20.7-24 \mathrm{~dB}$.

Normalized cross-correlations between all pairs of antennas were measured and the raw data processed according to [9]. This included the offset correction using all zero/all one correlations, quadrature error correction using each receivers IQ self-correlation and in-phase correction using the correlated noise injection through noise distribution network. The results are shown in Fig. 8 in which the measurements are given in a complex plot. Each cloud corresponds to a different baseline and each dot in the cloud is a single acquisition with an integration time of $1.2 \mathrm{~s}$. It can be clearly seen that the amplitude of the normalized correlation for the nearest antennas $(1-2,2-3$, and 3-4) are of the order of $50 \times 10^{-4}$ which is consistent with the simulations described in Section II-D. The isolator used in the receivers was the same that was used in the simulation.

\section{CONCLUSION}

A general analysis of correlation and total power radiometer front-ends must include mismatch between components and full noise characterization of individual receivers. While in total power radiometers most nonideal effects can be negligible, the effect of mismatch in correlation radiometers can be significant and results in visibility errors. This makes, for example, that when a baseline is placed inside an anechoic chamber, there exists a residual correlation proportional to the complex antenna coupling parameter and due to imperfect

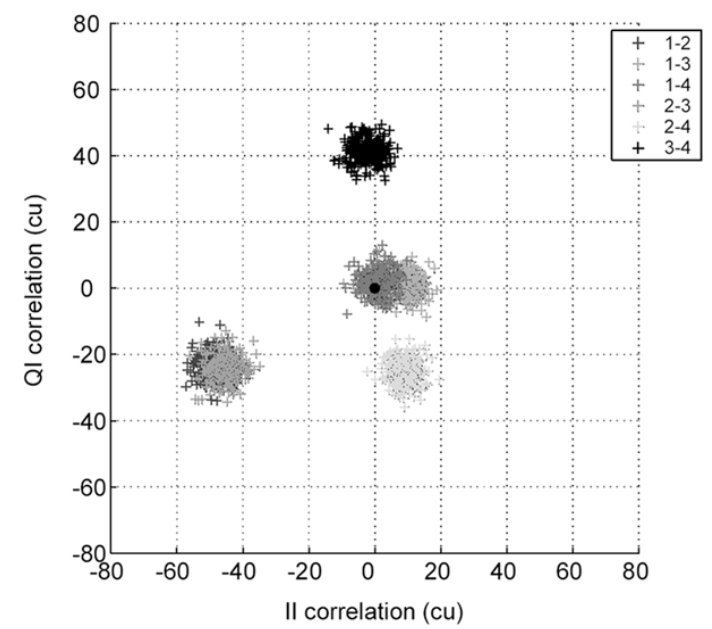

Fig. 8. Measured normalized correlations ( $1 \mathrm{cu}=10^{-4}$ units) of several baselines inside an anechoic chamber.

input isolators. This situation and others can be analyzed with the general formulation presented in this paper, in which no approximations are made. Additionally, a simple formula for estimating this residual offset when the isolators are of reasonable quality is also presented. The formula shows that the offset can range between zero and a maximum depending on the phases of different terms and that this maximum depends on the antenna coupling and the receiver input reflection coefficient. The theoretical results have been validated with measurements of cross-correlation between receivers at different distances inside an anechoic chamber.

\section{APPENDIX}

\section{NoISE WAVES AND EQuivalent NoISE TEMPERATURES}

The total output waves of a noisy two-port characterized by the S-parameters are given by [1]

$$
b_{1}=S_{11} a_{1}+S_{12} a_{2}+c_{1} \quad b_{2}=S_{21} a_{1}+S_{22} a_{2}+c_{2}
$$

where $a_{1}$ and $a_{2}$ are the external input waves and $c_{1}$ and $c_{2}$ the noise waves generated by the network itself. They are defined as the output waves of the network when both ports are connected to the reference impedance at absolute zero temperature, or equivalently when $a_{1}=a_{2}=0$. The noise correlation matrix of a network is defined as

$$
\mathbf{C}_{\mathbf{s}}=\left[\begin{array}{ll}
\overline{\left|c_{1}\right|^{2}} & \overline{c_{1} c_{2}{ }^{*}} \\
c_{2} c_{1}{ }^{*} & \overline{\left|c_{2}\right|^{2}}
\end{array}\right]
$$

which, for passive two-port networks at constant physical temperature $T_{\mathrm{ph}}$ turns out to be given by

$$
\mathbf{C}_{\mathbf{s}}=k T_{\mathrm{ph}}\left(\mathbf{I}-\mathbf{S S}^{\dagger}\right)
$$

where $k$ is the Boltzmann constant, the dagger indicates hermitian transpose and $\mathbf{I}$ is the identity matrix. The above formula is known as the Bosma theorem [10].

To analyze the noise of front-ends formed by cascaded twoports, it is convenient to introduce a noise description based on equivalent temperatures referred to the input port. The following definitions, already proposed in [3, eqs. (6)-(8)], are used:

$$
k T_{r}=\overline{\left|c_{1}\right|^{2}} \quad k T_{c}=\frac{\overline{c_{1} c_{2} *}}{S_{21}^{*}} \quad k T_{R}=\frac{\overline{\left|c_{2}\right|^{2}}}{\left|S_{21}\right|^{2}} .
$$


Note that $T_{r}$ and $T_{R}$ are always real while $T_{c}$ is complex in general. It is important to point out that $T_{R}$ is exactly the standard definition of equivalent noise temperature for zero source reflection coefficient, as indicated in [1, eq. (10)]. In (12) of this reference a general equation for the equivalent noise temperature for arbitrary source reflection coefficient $\left(\Gamma_{a}\right)$ is given. If the definitions (37) are substituted in this result, the following expression is found:

$$
\begin{aligned}
T_{R}\left(\Gamma_{a}\right)=\frac{\left|\Gamma_{a}\right|^{2}}{1-\left|\Gamma_{a}\right|^{2}} & T_{r}+\frac{\left|1-S_{11} \Gamma_{a}\right|^{2}}{1-\left|\Gamma_{a}\right|^{2}} T_{R} \\
& +2 \frac{\left|1-S_{11} \Gamma_{a}\right|^{2}}{1-\left|\Gamma_{a}\right|^{2}} \Re e\left[\frac{\Gamma_{a} T_{c}}{1-S_{11} \Gamma_{a}}\right] .
\end{aligned}
$$

This result is used to prove (19). Inserting (18) into (17) and identifying with (38) gives

$$
T_{\mathrm{sys}}=\frac{T_{a}+T_{R}\left(\Gamma_{a}\right)\left(1-\left|\Gamma_{a}\right|^{2}\right)}{\left|1-S_{11} \Gamma_{a}\right|^{2}} .
$$

On the other hand, the available power gain of a two-port network is defined as [11, eq. 3.2.4])

$$
G_{\mathrm{av}}\left(\Gamma_{a}\right)=\frac{\left|S_{21}\right|^{2}\left(1-\left|\Gamma_{a}\right|^{2}\right)}{\left|1-S_{11} \Gamma_{a}\right|^{2}\left(1-\left|\Gamma_{\text {out }}\left(\Gamma_{a}\right)\right|^{2}\right)}
$$

where $\Gamma_{\text {out }}\left(\Gamma_{a}\right)$ is the output reflection coefficient when the source reflection coefficient is $\Gamma_{a}$. After introducing (40) in (39), (19) follows immediately.

For the case of a passive network at constant temperature, using (36), the equivalent temperatures become

$$
\begin{aligned}
T_{r} & =T_{\mathrm{ph}}\left(1-\left|S_{11}\right|^{2}-\left|S_{12}\right|^{2}\right) \\
T_{c} & =-T_{\mathrm{ph}}\left(S_{11}+\frac{S_{12} S_{22}^{*}}{S_{21}^{*}}\right) \\
T_{R} & =T_{\mathrm{ph}} \frac{1-\left|S_{22}\right|^{2}-\left|S_{21}\right|^{2}}{\left|S_{21}\right|^{2}} \\
& =T_{\mathrm{ph}}\left(\frac{1-\left|S_{22}\right|^{2}}{\left|S_{21}\right|^{2}}-1\right) .
\end{aligned}
$$

Taking into account that $\left|S_{21}\right|^{2} /\left(1-\left|S_{22}\right|^{2}\right)$ is the available power gain of the network (40) for zero source reflection coefficient, this last equation is recognized as the standard formula for noise temperature of a passive network $T_{R}=T_{\mathrm{ph}}(L-1)$ where $L$ is the inverse of the available power gain.

Finally, the noise-equivalent temperatures of a cascade of two networks (labeled $a$ and $b$ ) can be computed using the techniques described in [1]. The following formulas are found:

$$
\begin{aligned}
T_{r_{T}=}= & T_{r_{a}}+\frac{\left|S_{a_{12}}\right|^{2}}{|D|^{2}} T_{r_{b}}+\left|\frac{S_{a_{12}} S_{a_{21}} S_{b_{11}}}{D}\right|^{2} T_{R_{a}} \\
& +2 \Re e\left[\frac{S_{a_{12}} S_{a_{21}} S_{b_{11}}}{D} T_{c_{a}}^{*}\right] \\
T_{c_{T}}= & T_{c_{a}}+\frac{S_{a_{12}} D^{*}}{S_{a_{21}}^{*} D} T_{c_{b}}+\frac{S_{a_{12}} S_{a_{21}} S_{b_{11}}}{D} T_{R_{a}}+\frac{S_{a_{12}} S_{a_{22}}^{*}}{S_{a_{21}}^{*} D} T_{r_{b}} \\
T_{R_{T}=}= & T_{R_{a}}+\frac{T_{R_{b}}|D|^{2}+2|D|^{2} \Re e\left[\frac{S_{a_{22}} T_{c_{b}}}{D}\right]+\left|S_{a_{22}}\right|^{2} T_{r_{b}}}{\left|S_{a_{21}}\right|^{2}}
\end{aligned}
$$

where $D=1-S_{b_{11}} S_{a_{22}}$. The last equation is recognized as the Friis formula since the second term is the ratio between the equivalent noise temperature of the network $b$ when its input is connected to a reflection coefficient equal to $S_{a_{22}}$ (see [1, eq. (12)]) and the available power gain of network $a$ when its input is connected to a the reference impedance.

\section{ACKNOWLEDGMENT}

The LNA data was provided by MIER Comunicaciones S.A.

\section{REFERENCES}

[1] S. W. Wedge and D. B. Rutledge, "Wave techniques for noise modeling and measurement," IEEE Trans. Microw. Theory Tech., vol. 40, no. 11, pp. 2004-2012, Nov. 1992.

[2] I. Corbella, N. Duffo, M. Vall-llossera, A. Camps, and F. Torres, "The visibility function in interferometric aperture synthesis radiometry," IEEE Trans. Geosci. Remote Sens., vol. 42, no. 8, pp. 1677-1682, Aug. 2004.

[3] I. Corbella, A. Camps, F. Torres, and J. Bará, "Analysis of noise injection networks for interferometric radiometer calibration," IEEE Trans. Microw. Theory Tech., vol. 48, no. 4, pp. 545-552, Apr. 2000.

[4] S. W. Wedge and D. B. Rutledge, "Noise waves and passive linear multiports," IEEE Microw. Guided Wave Lett., vol. 1, no. 5, pp. 117-119, May 1991.

[5] M. Martín-Neira and J. M. Goutoule, "A two-dimensional aperture-synthesis radiometer for soil moisture and ocean salinity observations," ESA Bull., no. 92, pp. 95-104, Nov. 1997.

[6] P. Silvestrin, M. Berger, Y. Kerr, and J. Font, "ESA's second earth explorer opportunity mission: The Soil Moisture and Ocean Salinity mission-SMOS," IEEE Geosci. Remote Sens. Newslett., no. 3, pp. 11-14, Mar. 2001.

[7] F. T. Ulaby, R. K. Moore, and A. K. Fung, Microwave Remote Sensing. Active and Passive. Norwell, MA: Artech House, 1981, vol. I, Fundamentals and Radiometry.

[8] K. Rautiainen, R. Butora, T. Auer, J. Kettunen, J. Kainulainen, I. Mononen, D. Beltrami, and M. Hallikainen, "Development of airborne aperture synthetic radiometer (HUT-2D)," in Proc. IGARSS, vol. 1, Toulouse, France, Jul. 21-25, 2003, pp. 1232-1234.

[9] I. Corbella, F. Torres, A. Camps, A. Colliander, M. Martín-Neira, S. Ribó, K. Rautiainen, N. Duffo, and M. Vall-llossera, "MIRAS end-to-end calibration: Application to SMOS L1 processor," IEEE Trans. Geosci. Remote Sens., vol. 43, no. 5, pp. 1126-1134, May 2005.

[10] H. Bosma, "On the theory of linear noisy systems," Ph.D. thesis, Technische Hogeschool Te Eindhoven, Eindhoven, The Nederlands, 1967.

[11] G. Gonzalez, Microwave Transistor Amplifiers. Analysis and Design, 2nd ed. Upper Saddle River, NJ: Prentice-Hall, 1997.

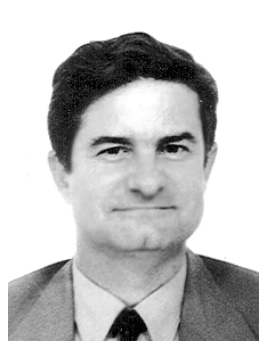

Ignasi Corbella (M'99) received the Telecommunications Engineering and Dr.Eng. degrees, both from Universitat Politècnica de Catalunya (UPC), Barcelona, Spain, in 1977 and 1983, respectively.

In 1976, he joined the School of Telecommunication Engineering in UPC as a Research Assistant in the Microwave Laboratory, where he worked on passive microwave integrated circuit design and characterization. During 1979, he worked at ThomsonCSF, Paris, France, on microwave oscillators design. In 1982, he became an Assistant Professor at UPC, an Associate Professor in 1986, and a Full Professor in 1993. He is currently teaching microwaves at the undergraduate level in UPC and has designed and taught graduate courses on nonlinear microwave circuits. During the school year 1998-1999, he worked at NOAA/Environmental Technology Laboratory, Boulder, CO, as a Guest Researcher, developing methods for radiometer calibration and data analysis. His research work in the Department of Signal Theory and Communications, UPC includes microwave airborne and satellite radiometry and microwave system design. 


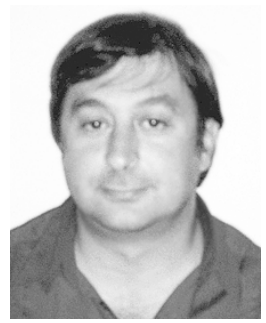

Francesc Torres (M'96) received the Ingeniero and Doctor Ingeniero degrees in telecommunication engineering from the Polytechnic University of Catalonia (UPC), Barcelona, Spain, in 1988 and 1992, respectively

In 1988-1989, he was a Research Assistant in the RF System Division, European Space Agency, Noordwijk, The Netherlands, devoted to microwave device testing and characterization. In 1989, he joined the Antenna-Microwave-Radar group, UPC, where he is currently an Associate Professor. His main research interests are focused on the design and testing of microwave systems and subsystems. He is currently engaged in research on interferometric radiometers devoted to earth observation.

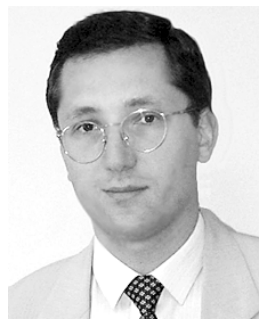

Adriano Camps (S'91-A'97-M'00-SM'02) was born in Barcelona, Spain, in 1969. He received the Telecommunications Engineering degree and the $\mathrm{Ph} . \mathrm{D}$. degree in telecommunications engineering in 1992 and 1996, respectively, both from the Polytechnic University of Catalonia (UPC), Barcelona, Spain.

From 1991 to 1992, he was with the ENS des Télécommunications de Bretagne, Bretagne, France, with an Erasmus Fellowship. In 1993, he joined the Electromagnetics and Photonics Engineering group, at the Department of Signal Theory and Communications, UPC, as an Assistant Professor, and since 1997 as an Associate Professor. In 1999, he was on sabbatical leave at the Microwave Remote Sensing Laboratory, University of Massachusetts, Amherst. His research interests are microwave remote sensing, with special emphasis in microwave radiometry by aperture synthesis techniques. He has performed numerous studies within the frame of European Space Agency SMOS Earth Explorer Mission. He is an Associate Editor of Radio Science.

Dr. Camps received the second national award of university studies in 1993, the INDRA award of the Spanish Association of Telecommunication Engineering to the best Ph.D. in 1997, the extraordinary Ph.D. award at the Universitat Politècnica de Catalunya in 1999, the First Duran Farell Award and the Ciudad de Barcelona Award, in 2000 and 2001, respectively, both for Technology Transfer, in 2002, the Research Distinction of the Generalitat de Catalunya for contributions to microwave passive remote sensing, in 2003, the Premi Nacional de Telecomunicacions (Generalitat de Catalunya) with the members of the Electromagnetics and Photonics Engineering group, and in 2004 a EURYI (European Young Investigator) Award. Also, as a member of the Microwave Radiometry Group at UPC, he received in 2000, 2001, and 2004 the 1st Duran Farell and the Ciudad de Barcelona Awards for Technology Transfeer, and the Salvà i Campillo Award of the Telecommunications (Engineering College of Catalonia) to the most innovative research project. He was Chair of Cal ' 01 . He is editor of the IEEE Geoscience and Remote Sensing Newsletter and President-Founder of the IEEE Geoscience and Remote Sensing Society Spain Chapter.

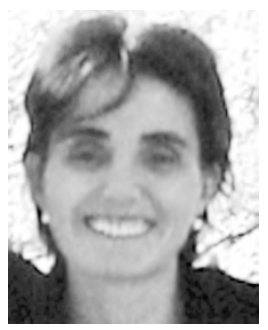

Núria Duffo (S'91-M'99) received the Telecommunication Engineer degree from the Polytechnic University of Catalonia (UPC), Barcelona, Spain, and the Doctor in Telecommunication Engineering from UPC, in 1990 and 1996, respectively.

Since 1997, she has been an Associate Professor at UPC. Her current research interests are numerical methods in electromagnetics, microwave radiometry, antenna analysis, and design.

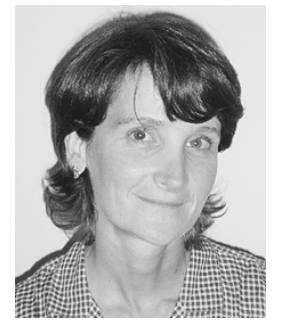

Mercè Vall-llossera (M'99) received the Senior Telecommunication Engineer and the Doctor Telecommunication Engineering degrees in 1990 and 1994, respectively, both from the Polytechnic University of Catalonia (UPC), Barcelona, Spain.

She has been lecturing and doing research at the Department of Signal Theory and Communications, UPC from 1990 until 1997 as an Assistant Professor and from 1997 until present as an Associate Professor. She spent a sabbatical year in Montreal with the scholarship of the "Programme Québécois de Bourses d'excellence" (1996-1997): "Stages de Formation postdoctorale au Québec pour jeunes diplômés étrangers.” Her research interests include numerical methods in electromagnetism, microwave radiometry, antenna analysis, and design. Currently, her research is mainly related to the study of numerical methods applied to the sea surface emissivity and their characterization at L-band and the MIRAS/SMOS project.

Dr. Vall-llossera, along with the other members of the radiometry group at UPC, was awarded the "9th Edition of the Salvà i Campillo Award" in 2004, the "Primer Premio Duran Farell de Investigación Tecnológica" in 2002, and the "Primer Premio Ciutat de Barcelona d'Investigació Tecnològica" in 2001.

Kimmo Rautiainen was born 1969 in Säkylä, Finland. He received the M.Sc. degree from the Helsinki University of Technology (TKK), Espoo, Finland, in 1996.

He is currently a Research Scientist and Project Manager in the Laboratory of Space Technology, TKK. His research is focused on microwave radiometer systems, with emphasis on interferometric radiometers. He is currently responsible of technical matters on an ariborne two-dimensional interferometric radiometer.

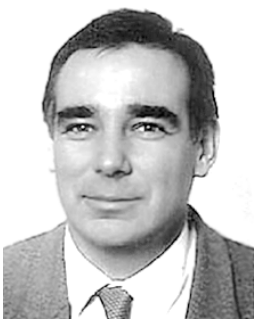

Manuel Martín-Neira (M'96) received the M.S. and $\mathrm{Ph} . \mathrm{D}$. degrees in telecommunication engineering in 1986 and 1996, respectively, from the School of Telecommunication Engineering, Polytechnical University of Catalonia, Catalonia, Spain.

From 1989 to 1992, he was was with GMV, Spanish firm, where he was responsible for several projects for the European Space Agency (ESA) related to global positioning satellite navigation with applications to precise landing and attitude determination. Since 1992, with ESA, he has been in charge of the radiometer activities within the Payload, Equipment, and Technology Section. During this period, he has been responsible for the predevelopment technology activities related to the Microwave Imaging Radiometer with Aperture Synthesis (MIRAS) project. MIRAS (L-band Microwave Imaging Radiometer with Aperture Synthesis) is the only payload onboard the Soil Moisture and Ocean Salinity mission (SMOS), ESA's second Earth Explorer Opportunity Mission. He is currently the SMOS Instrument Principal Engineer

Dr. Martín-Neira was awarded a Fellowship to work on radiometry at European Space Research and Technology Center (ESTEC), Noordwijk, The Netherlands, in 1988

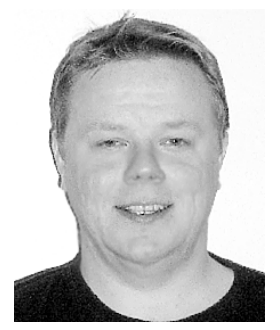

Andreas Colliander was born in 1976 in Imatra, Finland. He received the M.Sc. degree from the Helsinki University of Technology (TKK), Espoo, Finland, in 2002. He is currently pursuing the D.Sc. degree at TKK.

$\mathrm{He}$ is a Research Scientist and Project Manager in the Laboratory of Space Technology, TKK. He also has a research student position in the national Graduate School for Remote Sensing, which is supported by Finnish Ministry of Education and Academy of Finland. His research is focused on microwave radiometer systems, with emphasis on polarimetric and interferometric radiometers, and on theoretical simulation of rough surface backscattering. He has authored and coauthored 11 scientific publications on microwave remote sensing.

Mr. Colliander received the 2002 TKK Master's Thesis Award, an annual award for top five Master's thesis of TKK. 